\title{
Fitting Low-resolution Protein Structures into Cryo-EM Density Maps by Multi-objective Optimization of Global and Local Correlations
}

\author{
Biao Zhang ${ }^{1,2}$, Wenyi Zhang ${ }^{3}$, Robin Pearce ${ }^{2}$, Yang Zhang*,2, Hong-Bin Shen ${ }^{*, 1}$ \\ ${ }^{1}$ Institute of Image Processing and Pattern Recognition, Shanghai Jiao Tong University, \\ and Key Laboratory of System Control and Information Processing, Ministry of \\ Education of China, Shanghai, China \\ ${ }^{2}$ Department of Computational Medicine and Bioinformatics, \\ University of Michigan, Ann Arbor, MI 48109 USA \\ ${ }^{3}$ School of life Sciences, Westlake University, \\ Hangzhou, Zhejiang, 310024, China
}

\section{Supporting Information}

\section{Table of Content}

\section{Supporting Tables}

- Table S1. Summary of the resolutions for the 292 test proteins.

- Table S2. Summary of modeling results by I-TASSER structure prediction and the follow-up EM density map fitting methods on 7 proteins with experimentally determined density maps.

- Table S3. Summary of comparison results between each single objective fitting method and MOFIT on 292 test proteins.

\section{Supporting Figures}

- Figure S1. Histogram distribution of the simulated density map resolutions for the 292 benchmark test proteins.

- Figure S2. Performance of fitting native structures into density maps. The $\mathrm{x}$-axis represents the range of cRMSD values, while the $\mathrm{y}$-axis represents the corresponding counts numbers.

- Figure S3. The correlation coefficients $(C C)$ to the EM density map versus TM-score of the initial models for the 292 proteins after fitting. The dashed-dotted line divides the models into two regions with TM-scores below and above 0.5, where the solid lines represent the fitting results by linear regression for the samples in the two regions. The Pearson correlation 
coefficients between $C C$ and TM-score were 0.18 and 0.74 , respectively, for the two regions.

- Figure S4. An illustrative example to show the energy distribution of the nondominated particles of the Pareto set in energy space for target T0880.

- Figure S5. Illustrative head-to-head comparison between each single objective function. Each block represents the cRMSD value on the corresponding axis.

- Figure S6. Objective function values for one case (1efdN) during the PSO searching iterations: the E1, E2 and the E3 values at each iteration are shown in (a), (b) and (c), respectively.

- Figure S7. Illustration of the correlation between cRMSD and TM-score of each fitted model at different density map resolutions. (a) the Pearson correlation coefficient between cRMSD and TM-score is -0.61 for 5-6 $\AA$ maps, (b) -0.52 for $7-8 \AA$ maps, and (c) -0.50 for $9-10 \AA$ maps. 


\section{Supporting Tables}

Table S1. Summary of the resolutions for the 292 test proteins.

\begin{tabular}{|c|c|c|c|c|c|}
\hline Protein name & Resolution $(\AA)$ & Protein name & Resolution $(\AA)$ & Protein name & Resolution $(\AA)$ \\
\hline 1efdN_dom1 & 7 & 3a1iA_dom1 & 7 & 3wkuA_dom2 & 8 \\
\hline 1efdN_dom2 & 7 & 3a1iA_dom2 & 10 & 3zvmA_dom 1 & 7 \\
\hline 1g87B_dom1 & 9 & 3a45A_dom1 & 6 & 3zvmA_dom2 & 7 \\
\hline 1g87B_dom2 & 6 & 3a45A_dom2 & 8 & 4ap5A_dom1 & 5 \\
\hline 1gttA_dom1 & 9 & 3ajeA_dom2 & 6 & 4ap5A_dom2 & 6 \\
\hline 1iwaA_dom1 & 9 & 3aqkA_dom1 & 7 & 4axdA_dom1 & 7 \\
\hline 1iwaA_dom2 & 7 & 3aqkA_dom 2 & 7 & 4axdA_dom2 & 9 \\
\hline 1mkmB_dom 1 & 10 & 3arbA_dom1 & 5 & 4bfiB_dom 1 & 7 \\
\hline 1mkmB_dom2 & 7 & 3arbA_dom2 & 5 & 4bfiB_dom 2 & 10 \\
\hline 1pprM_dom1 & 9 & 3b2zF_dom 1 & 10 & 4c9zA_dom2 & 8 \\
\hline 1prrA_dom 1 & 5 & 3b2zF_dom 2 & 7 & 4cczA_dom1 & 5 \\
\hline 1q19A_dom1 & 6 & 3b7wA_dom1 & 6 & 4cczA_dom2 & 7 \\
\hline 1q19A_dom2 & 6 & 3b7wA_dom 2 & 6 & 4d0nB_dom1 & 8 \\
\hline 1qwrA_dom1 & 5 & 3bt3A_dom 1 & 6 & 4d0nB_dom2 & 8 \\
\hline 1qwrA_dom2 & 6 & 3bt3A_dom2 & 6 & 4dj3A_dom1 & 8 \\
\hline 1rh1A_dom1 & 5 & 3bu2A_dom2 & 6 & 4dj3A_dom2 & 7 \\
\hline 1rh1A_dom2 & 7 & 3c4tA_dom 1 & 9 & 4dqaA_dom1 & 5 \\
\hline 1rktA_dom1 & 9 & 3c4tA_dom 2 & 7 & 4dqaA_dom2 & 6 \\
\hline 1rktA_dom2 & 8 & 3craA_dom1 & 7 & 4eo3A_dom1 & 6 \\
\hline 1s61A_dom1 & 9 & 3craA_dom 2 & 10 & 4eo3A_dom2 & 6 \\
\hline 1s61A_dom2 & 7 & 3d30A_dom 1 & 6 & 4etxA_dom1 & 5 \\
\hline 1st0A_dom 2 & 9 & 3d30A_dom 2 & 9 & 4etxA_dom2 & 10 \\
\hline 1upsA_dom1 & 7 & 3e00D_dom 1 & 6 & 4fkcA_dom1 & 8 \\
\hline 1v8cA_dom2 & 7 & 3errA_dom1 & 7 & 4fkcA_dom2 & 5 \\
\hline 1w3aA_dom1 & 9 & 3errA_dom2 & 8 & 4fxkC_dom 1 & 8 \\
\hline 1w3aA_dom2 & 8 & 3g79A_dom1 & 5 & 4fxkC_dom2 & 8 \\
\hline 1wfxA_dom1 & 8 & 3g79A_dom 2 & 9 & 4g86A_dom1 & 9 \\
\hline 1wv3A_dom1 & 5 & 3hcsA_dom1 & 5 & 4g86A_dom2 & 7 \\
\hline 1wv3A_dom2 & 5 & 3hcsA_dom 2 & 7 & 4gbyA_dom1 & 10 \\
\hline 1x7pA_dom1 & 7 & 3hyiA_dom1 & 10 & 4gbyA_dom2 & 9 \\
\hline 1x7pA_dom2 & 9 & 3hyiA_dom2 & 10 & 4ggmX_dom 1 & 7 \\
\hline 1yiqA_dom1 & 10 & 3i2dA_dom1 & 9 & 4ggmX_dom2 & 6 \\
\hline 1yiqA_dom2 & 5 & 3i2dA_dom2 & 10 & 4gslA_dom1 & 5 \\
\hline 1zbuB_dom 1 & 9 & 3iam2_dom1 & 7 & 4gslA_dom2 & 10 \\
\hline 1zbuB_dom 2 & 9 & 3iam2_dom2 & 8 & 4gyjA_dom1 & 7 \\
\hline 1ze1A_dom1 & 5 & 3ifrA_dom1 & 5 & 4gyjA_dom2 & 6 \\
\hline 1ze1A_dom2 & 10 & 3ifrA_dom2 & 9 & 4h3tA_dom1 & 9 \\
\hline 2ablA_dom1 & 8 & 3isqA_dom1 & 8 & 4h3tA_dom2 & 10 \\
\hline
\end{tabular}




\begin{tabular}{|c|c|c|}
\hline 2ablA_dom2 & 10 & 3isqA_dom2 \\
\hline 2ahvA_dom 1 & 7 & 3j7aK_dom 1 \\
\hline 2ahvA_dom2 & 9 & 3j7aK_dom 2 \\
\hline 2bkpA_dom 1 & 8 & 3k1rA_dom 1 \\
\hline 2bkpA_dom 2 & 7 & 3k1rA_dom 2 \\
\hline 2c11A_dom1 & 10 & 3kh5A_dom 1 \\
\hline 2c1yA_dom1 & 8 & 3kh5A_dom 2 \\
\hline 2c1yA_dom2 & 9 & 3kjpA_dom 1 \\
\hline 2cxcA_dom1 & 10 & 3kjpA_dom2 \\
\hline 2cxcA_dom2 & 10 & 3kt1A_dom1 \\
\hline 2d1cA_dom 1 & 5 & 3kt1A_dom 2 \\
\hline 2d7iA_dom1 & 6 & 3kzwA_dom 1 \\
\hline 2d7iA_dom2 & 5 & 3kzwA_dom 2 \\
\hline 2e9xB_dom 1 & 8 & 31sgA_dom1 \\
\hline 2e9xB_dom 2 & 6 & 31sgA_dom2 \\
\hline 2evrA_dom1 & 9 & 3me4A_dom 1 \\
\hline 2evrA_dom2 & 5 & 3me4A_dom2 \\
\hline 2ew9A_dom1 & 8 & 3ml4C_dom 1 \\
\hline 2fd5A_dom 1 & 7 & $3 \mathrm{ml} 4 \mathrm{C} \_$dom 2 \\
\hline 2fd5A_dom2 & 8 & 3mzfA_dom1 \\
\hline 2gh8A_dom1 & 9 & 3mzfA_dom 2 \\
\hline 2gh8A_dom2 & 8 & 3njaB_dom1 \\
\hline 2gt1A_dom1 & 10 & 3njaB_dom2 \\
\hline 2gt1A_dom2 & 8 & 3nt8A_dom1 \\
\hline 2gzaC_dom1 & 5 & 3nt8A_dom2 \\
\hline 2gzaC_dom2 & 6 & 3o2gA_dom 2 \\
\hline 2hyxA_dom 1 & 7 & 3og5A_dom 1 \\
\hline 2ijd1_dom1 & 5 & 3og5A_dom 2 \\
\hline 2ijd1_dom2 & 8 & 3oh0A_dom1 \\
\hline 2iw2A_dom1 & 9 & 3oh0A_dom2 \\
\hline 2iw2A_dom2 & 9 & 3qavA_dom1 \\
\hline 2jz4A_dom2 & 6 & 3qavA_dom2 \\
\hline 2mbgA_dom 1 & 8 & 3qf4B_dom2 \\
\hline 2mbgA_dom 2 & 9 & 3qijiA_dom1 \\
\hline 2nsfA_dom1 & 7 & 3qijiA_dom2 \\
\hline 2nsfA_dom2 & 7 & 3qtdA_dom1 \\
\hline 2nykA_dom1 & 7 & 3qtdA_dom2 \\
\hline 2nykA_dom2 & 7 & 3r6bA_dom1 \\
\hline 2o6yA_dom1 & 10 & 3rh7A_dom 1 \\
\hline 206yA_dom2 & 10 & 3rh7A_dom2 \\
\hline 2outA_dom1 & 6 & 3sb4A_dom 1 \\
\hline 2owbA_dom1 & 10 & 3sb4A_dom 2 \\
\hline 2owbA_dom2 & 6 & 3ssoB_dom 2 \\
\hline
\end{tabular}

\begin{tabular}{|c|c|c|}
\hline 10 & 4hmoA_dom 1 & 9 \\
\hline 6 & 4hmoA_dom2 & 6 \\
\hline 10 & 4hsaC_dom 1 & 7 \\
\hline 10 & 4hwiB_dom2 & 10 \\
\hline 8 & 415gA_dom 1 & 10 \\
\hline 8 & 415gA_dom 2 & 10 \\
\hline 10 & 4lpqA_dom1 & 8 \\
\hline 9 & 4lpqA_dom2 & 9 \\
\hline 10 & $4 \mathrm{mspA} A$ dom 2 & 7 \\
\hline 7 & 4n06B_dom1 & 8 \\
\hline 10 & 4n06B_dom2 & 8 \\
\hline 9 & 4nj5A_dom1 & 10 \\
\hline 8 & 4nj5A_dom2 & 10 \\
\hline 8 & 4up9A_dom1 & 5 \\
\hline 9 & 4up9A_dom2 & 7 \\
\hline 9 & 4w7sA_dom 1 & 10 \\
\hline 7 & 4w7sA_dom 2 & 7 \\
\hline 6 & T0863_dom1 & 8 \\
\hline 10 & T0880_dom1 & 5 \\
\hline 6 & T0880_dom2 & 6 \\
\hline 10 & T0886_dom1 & 5 \\
\hline 7 & T0886_dom2 & 8 \\
\hline 7 & T0890_dom1 & 6 \\
\hline 7 & T0890_dom2 & 6 \\
\hline 8 & T0892_dom1 & 8 \\
\hline 10 & T0892_dom2 & 5 \\
\hline 5 & T0893_dom1 & 7 \\
\hline 6 & T0893_dom2 & 10 \\
\hline 5 & T0894_dom1 & 10 \\
\hline 8 & T0894_dom2 & 5 \\
\hline 10 & T0896_dom1 & 7 \\
\hline 5 & T0896_dom2 & 10 \\
\hline 5 & T0897_dom1 & 5 \\
\hline 10 & T0897_dom2 & 8 \\
\hline 8 & T0898_dom1 & 8 \\
\hline 10 & T0898_dom2 & 10 \\
\hline 7 & T0912_dom1 & 9 \\
\hline 10 & T0914_dom1 & 5 \\
\hline 6 & T0914_dom2 & 7 \\
\hline 8 & T0918_dom1 & 9 \\
\hline 8 & T0918_dom2 & 8 \\
\hline 5 & T0920_dom1 & 8 \\
\hline 8 & T0920_dom2 & 9 \\
\hline
\end{tabular}




\begin{tabular}{|l|c|c|c|c|c|} 
2qp2A_dom1 & 6 & 3swjA_dom1 & 7 & T0942_dom1 & 6 \\
2qp2A_dom2 & 10 & 3swjA_dom2 & 7 & T1008 & 5 \\
2qygA_dom1 & 10 & 3t7jA_dom1 & 9 & T0998 & 7 \\
2qygA_dom2 & 7 & 3t7jA_dom2 & 6 & T0991 & 8 \\
2r5wB_dom1 & 6 & 3u07C_dom1 & 8 & T0980s1 & 6 \\
2r5wB_dom2 & 8 & 3u07C_dom2 & 10 & T0953s1 & 7 \\
2r8vA_dom2 & 7 & 3u0oB_dom1 & 8 & T0953s2 & 9 \\
2v42A_dom1 & 9 & 3u0oB_dom2 & 9 & T0986s2 & 5 \\
2w2gA_dom1 & 8 & 3ub1D_dom1 & 9 & T1015s1 & 8 \\
2w5fB_dom1 & 5 & 3ub1D_dom2 & 8 & T1015s2 & 9 \\
2wkxA_dom2 & 8 & 3uitD_dom1 & 7 & T0975 & 6 \\
2x7iA_dom1 & 5 & 3uitD_dom2 & 10 & T0968s2 & 8 \\
2x7iA_dom2 & 9 & 3uo3A_dom1 & 6 & T1019S1 & 5 \\
2x8kC_dom1 & 10 & 3uo3A_dom2 & 8 & T0955 & 9 \\
2x8kC_dom2 & 7 & 3vr8B_dom1 & 6 & T0992 & 7 \\
2yilA_dom1 & 9 & 3vr8B_dom2 & 10 & & \\
2yilA_dom2 & 10 & 3wkuA_dom1 & 8 & &
\end{tabular}

Note: Txxx represents the target from CASP experiment case. 
Table S2. Summary of modeling results by I-TASSER structure prediction and the follow-up EM density map fitting methods on 7 proteins with experimentally determined density maps.

\begin{tabular}{|c|c|c|c|c|c|}
\hline \multirow{2}{*}{ Protein Name } & \multirow{2}{*}{ Methods } & \multirow{2}{*}{$\begin{array}{l}\text { Resolution } \\
\text { (A) }\end{array}$} & \multicolumn{2}{|c|}{ Predicted structure } & \multirow{2}{*}{$\begin{array}{c}\text { Fitted } \\
\text { structure } \\
\text { cRMSD }\end{array}$} \\
\hline & & & TM-score & RMSD & \\
\hline \multirow{4}{*}{ EMD20239_dom1 } & ADP_EM & \multirow{4}{*}{3.1} & \multirow{4}{*}{0.734} & \multirow{4}{*}{$\begin{array}{c}14.09 \\
\AA\end{array}$} & $1.57 \AA$ \\
\hline & Situs & & & & $1.81 \AA$ \\
\hline & PowerFit & & & & $2.74 \AA$ \\
\hline & MOFIT & & & & $0.59 \AA$ \\
\hline \multirow{4}{*}{ EMD21923_dom2 } & ADP_EM & \multirow{4}{*}{3.3} & \multirow{4}{*}{0.756} & \multirow{4}{*}{$9.60 \AA$} & $1.93 \AA$ \\
\hline & Situs & & & & $1.92 \AA$ \\
\hline & PowerFit & & & & $3.78 \AA$ \\
\hline & MOFIT & & & & $1.82 \AA$ \\
\hline \multirow{4}{*}{ EMD21040_dom1 } & ADP_EM & \multirow{4}{*}{3.8} & \multirow{4}{*}{0.719} & \multirow{4}{*}{$5.35 \AA$} & $6.46 \AA$ \\
\hline & Situs & & & & $2.84 \AA$ \\
\hline & PowerFit & & & & $4.96 \AA$ \\
\hline & MOFIT & & & & $2.58 \AA$ \\
\hline \multirow{4}{*}{ EMD6847_dom1 } & ADP_EM & \multirow{4}{*}{4.4} & \multirow{4}{*}{0.811} & \multirow{4}{*}{$7.43 \AA$} & $0.96 \AA$ \\
\hline & Situs & & & & $0.86 \AA$ \\
\hline & PowerFit & & & & $1.36 \AA$ \\
\hline & MOFIT & & & & $0.86 \AA$ \\
\hline \multirow{4}{*}{ EMD6708_dom2 } & ADP_EM & \multirow{4}{*}{3.9} & \multirow{4}{*}{0.540} & \multirow{4}{*}{$7.02 \AA$} & $13.62 \AA$ \\
\hline & Situs & & & & $18.43 \AA$ \\
\hline & PowerFit & & & & $11.46 \AA$ \\
\hline & MOFIT & & & & $2.20 \AA$ \\
\hline \multirow{4}{*}{ EMD7454_dom2 } & ADP_EM & \multirow{4}{*}{6.72} & \multirow{4}{*}{0.666} & \multirow{4}{*}{$4.65 \AA$} & $1.60 \AA$ \\
\hline & Situs & & & & $1.74 \AA$ \\
\hline & PowerFit & & & & $1.88 \AA$ \\
\hline & MOFIT & & & & $0.86 \AA$ \\
\hline \multirow{4}{*}{ EMD2221_dom1 } & ADP_EM & \multirow{4}{*}{8.4} & & & $0.84 \AA$ \\
\hline & Situs & & & & $0.70 \AA$ \\
\hline & PowerFit & & 0.109 & $4.04 \mathrm{~A}$ & $1.75 \AA$ \\
\hline & MOFIT & & & & $1.58 \AA$ \\
\hline
\end{tabular}


Table S3. Summary of comparison results between each single objective fitting method and MOFIT on 292 test proteins.

\begin{tabular}{|c|c|c|c|}
\hline Method & Average cRMSD & Median & P value \\
\hline E1 & 4.03 & 1.29 & $1.65 \mathrm{E}-06$ \\
\hline E2 & 5.25 & 1.85 & $6.36 \mathrm{E}-08$ \\
\hline E3 & 6.41 & 1.88 & $6.44 \mathrm{E}-11$ \\
\hline MOFIT & 2.46 & 0.75 & -- \\
\hline
\end{tabular}




\section{Supporting Figures}

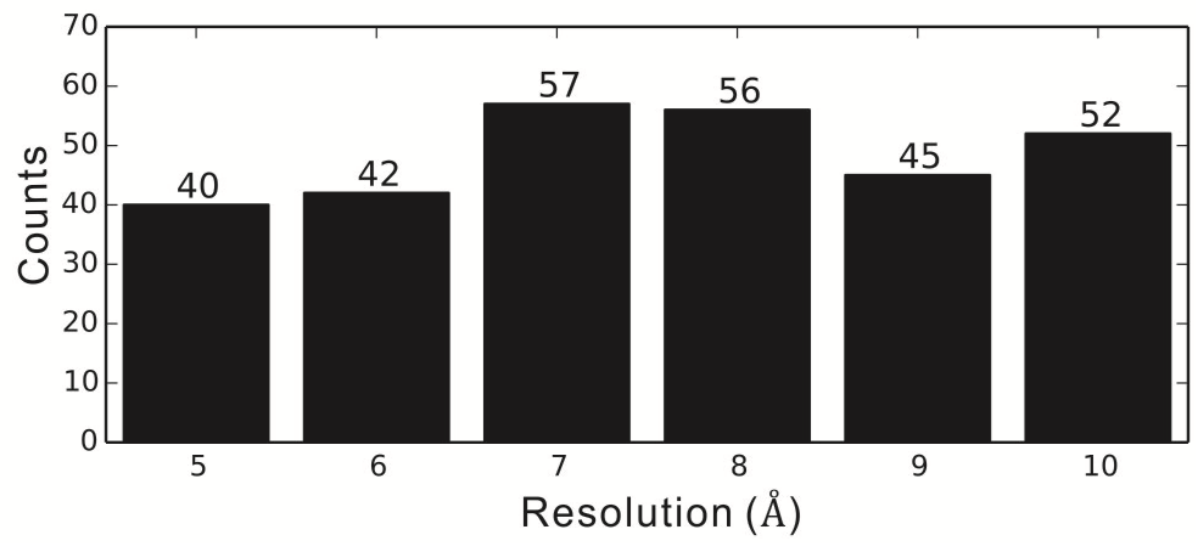

Figure S1. Histogram distribution of the simulated density map resolutions for the 292 benchmark test proteins. 


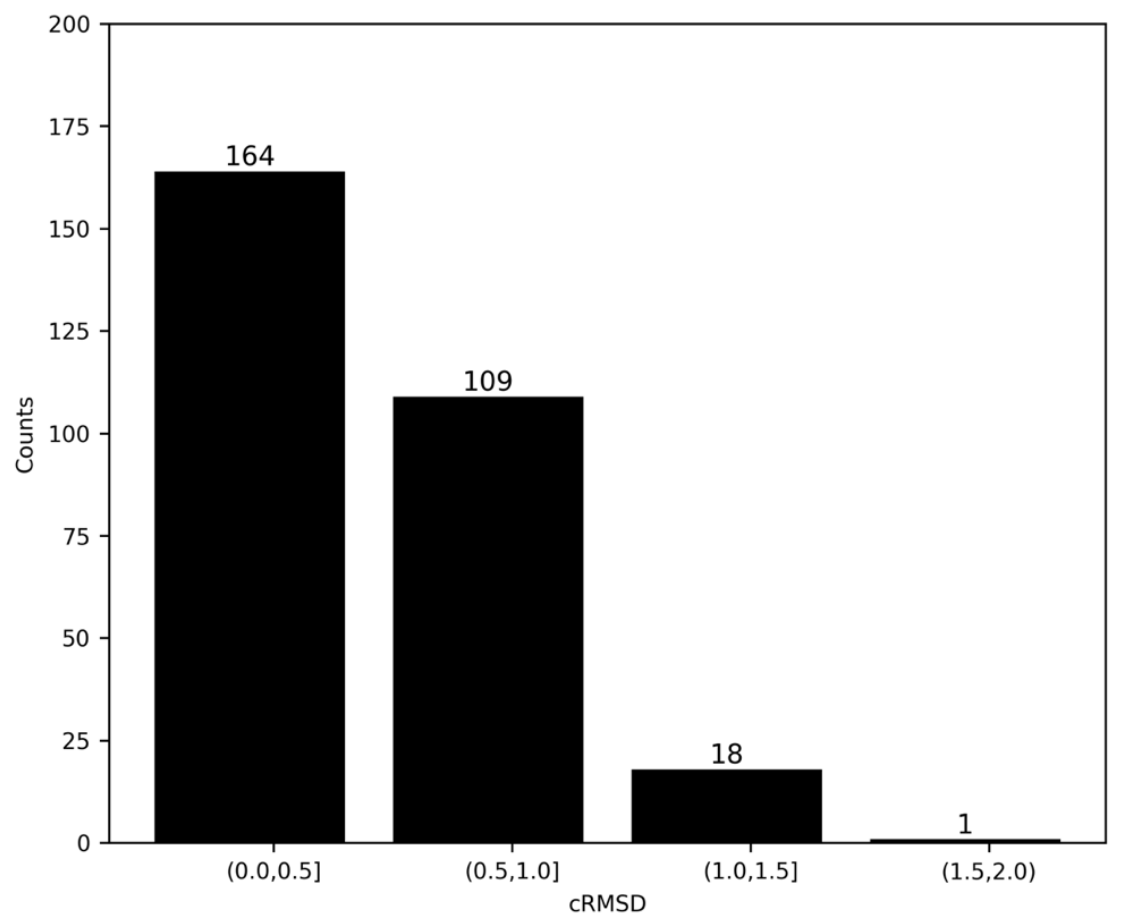

Figure S2. Performance of fitting native structures into density maps. The $\mathrm{x}$-axis represents the range of cRMSD values, while the y-axis represents the corresponding counts numbers. 


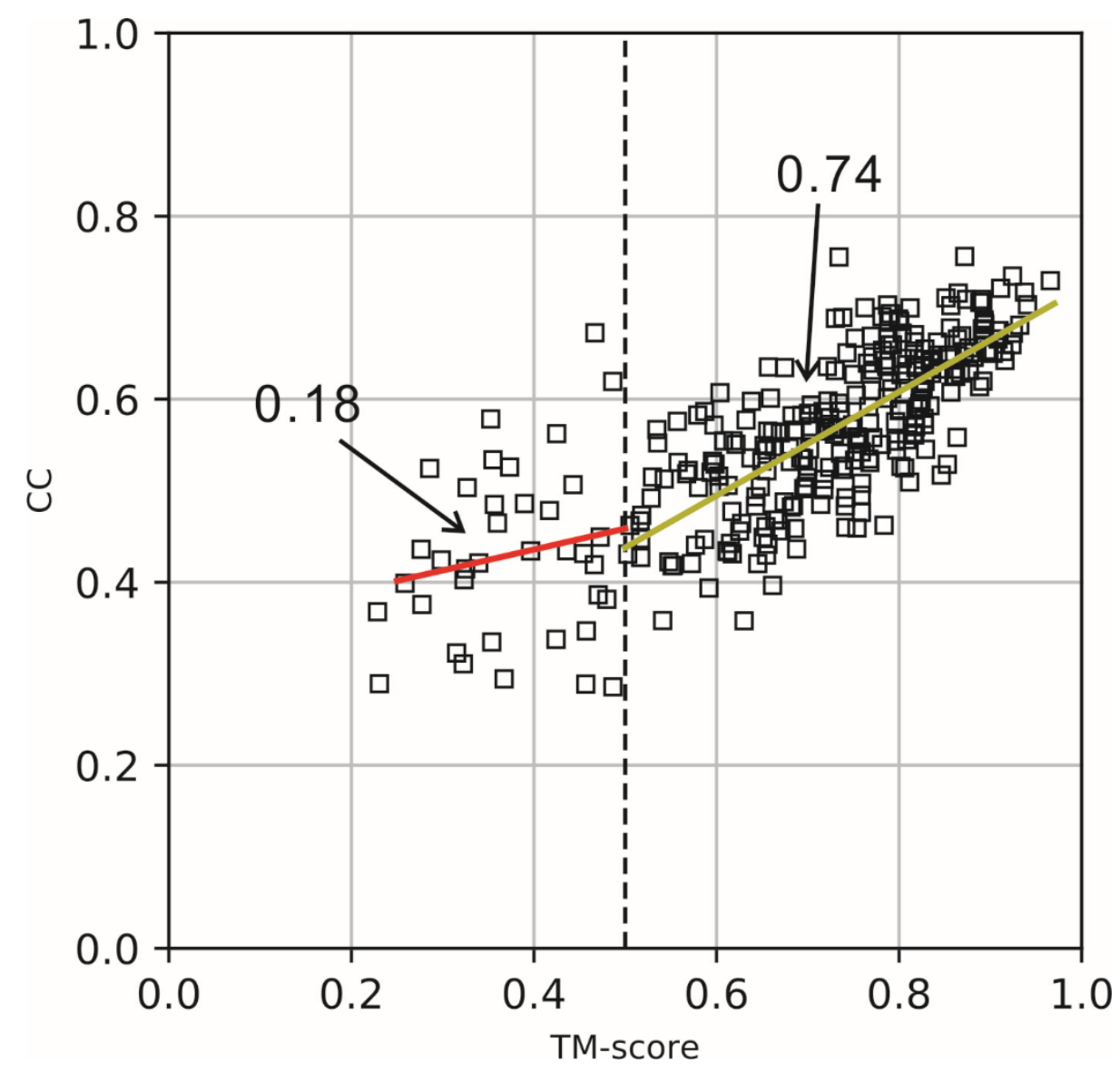

Figure S3. The correlation coefficients $(C C)$ to the EM density map versus TM-score of the initial models for the 292 proteins after fitting. The dashed-dotted line divides the models into two regions with TM-scores below and above 0.5 , where the solid lines represent the fitting results by linear regression for the samples in the two regions. The Pearson correlation coefficients between $C C$ and TM-score were 0.18 and 0.74 , respectively, for the two regions. 


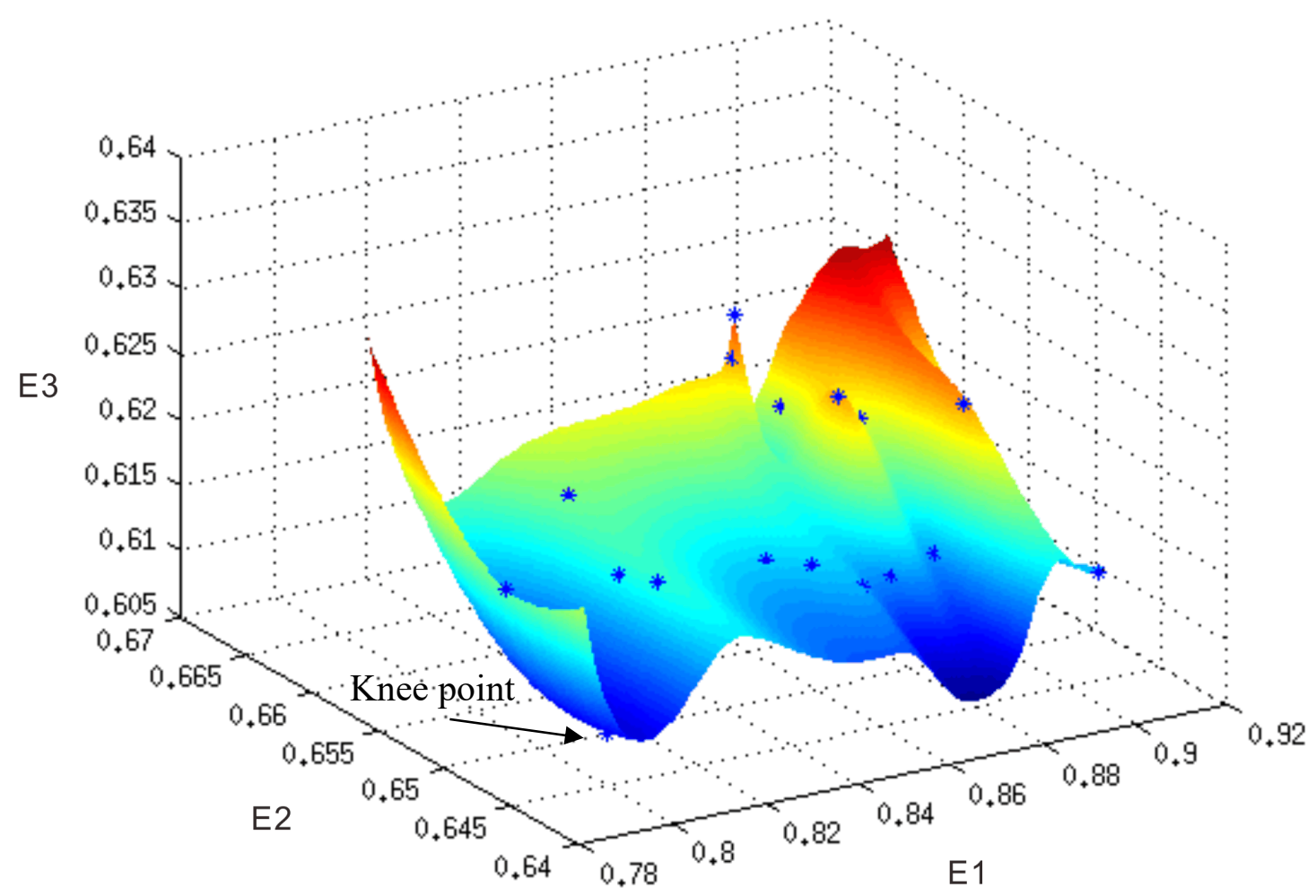

Figure S4. An illustrative example to show the energy distribution of the nondominated particles of the Pareto set in energy space for target T0880. 
(A)

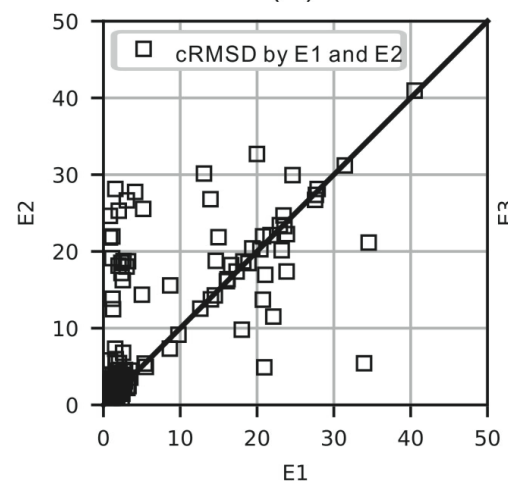

(B)

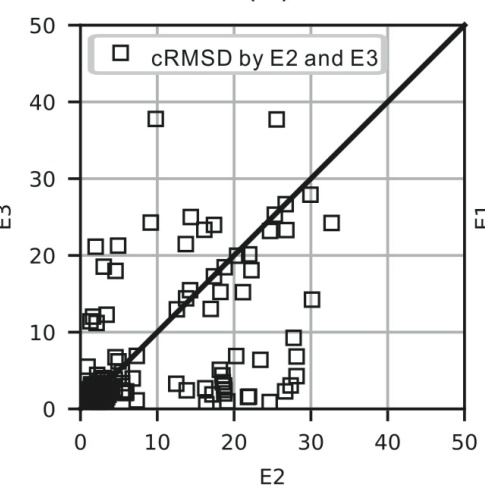

(C)

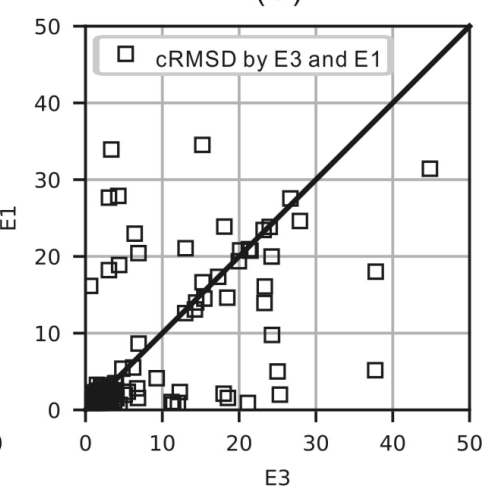

Figure S5. Illustrative head-to-head comparison between each single objective function. Each block represents the cRMSD value on the corresponding axis. 

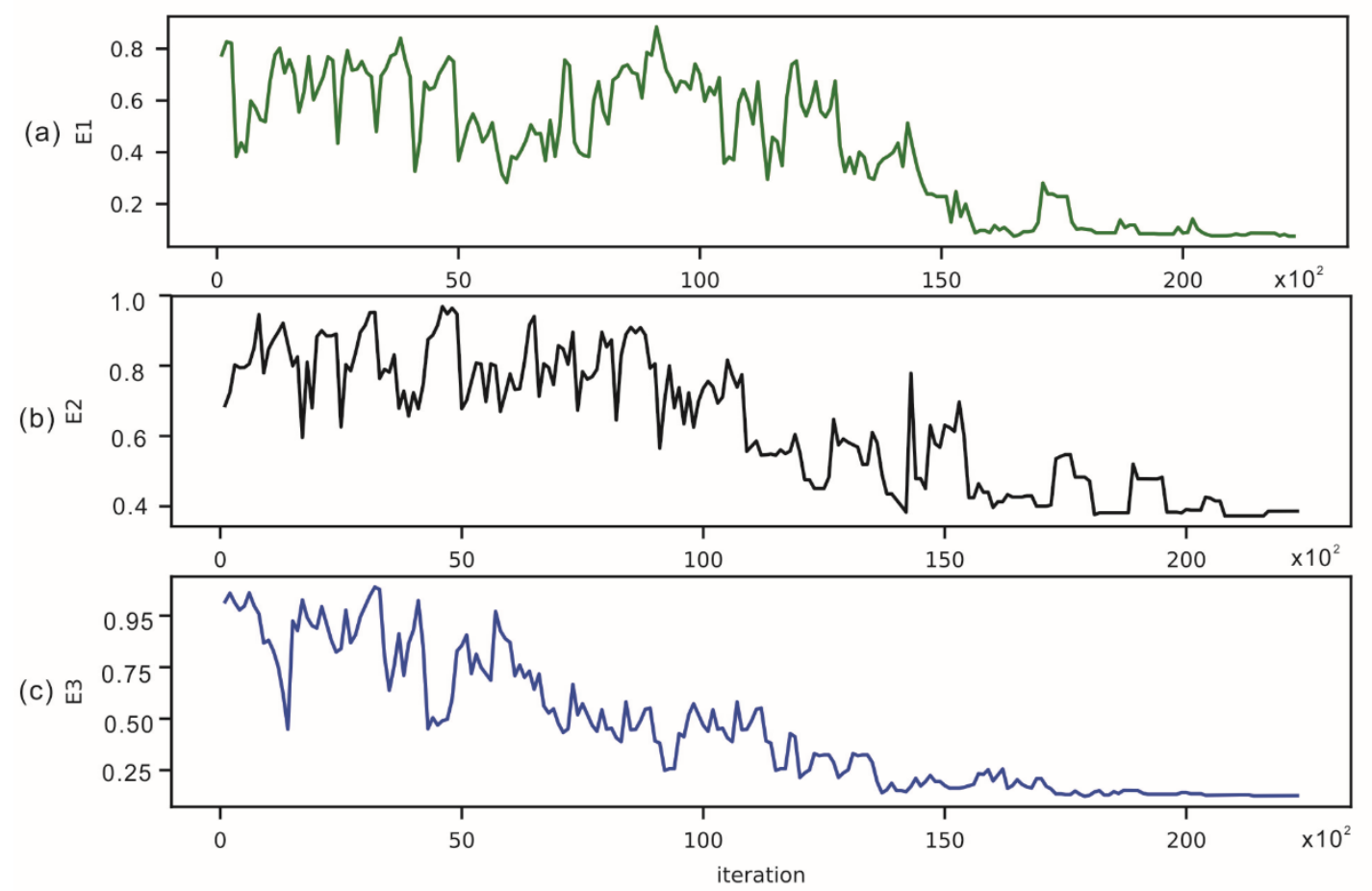

Figure S6. Objective function values for one case (1efdN) during the PSO searching iterations: the E1, E2 and the E3 values at each iteration are shown in (a), (b) and (c), respectively. 
(a)

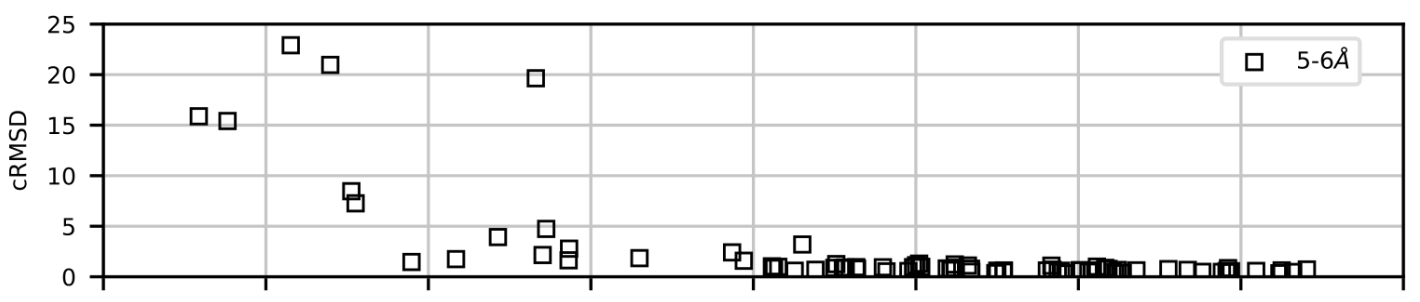

(b)

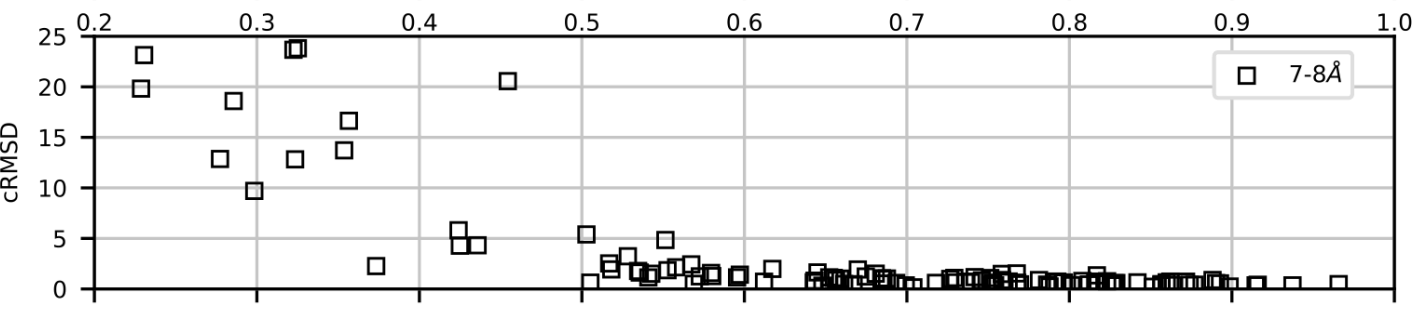

(c)

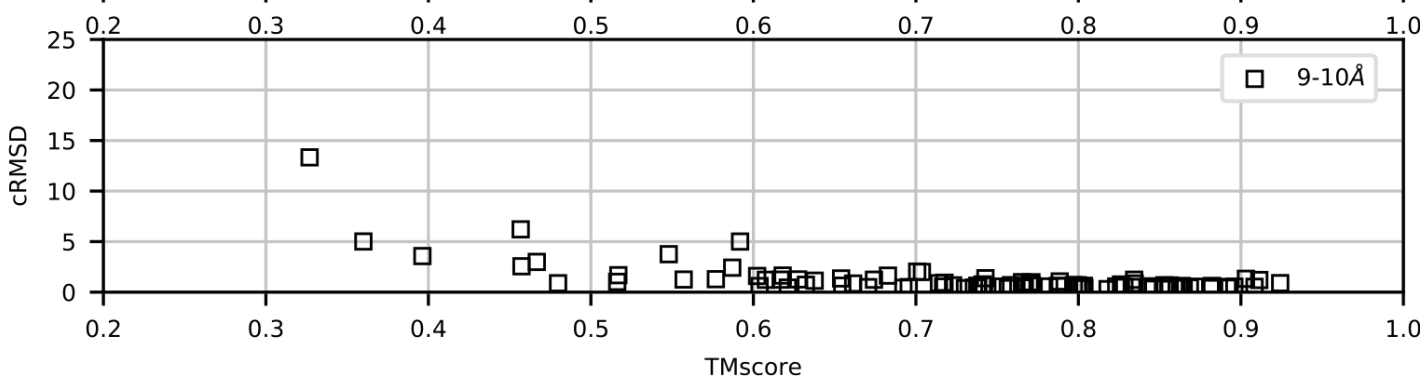

Figure S7. Illustration of the correlation between cRMSD and TM-score of each fitted model at different density map resolutions. (a) the Pearson correlation coefficient between cRMSD and TM-score is -0.61 for 5-6 $\AA$ maps, (b) - 0.52 for 7-8 $\AA$ maps, and (c) -0.50 for $9-10 \AA$ maps. 